\title{
Capacitance of the Double Layer at Polycrystalline Au-Ag Electrodes: Influence of Preparation Technique and Specific Anion Adsorption
}

\author{
A. Hammadi* and M. Berd \\ Département de Physique, Faculté des Sciences, Université Mentouri, \\ 25000 Constantine, Algérie
}

Received 12 February 2006; accepted 19 July 2007

\begin{abstract}
We present electrochemical impedance spectra made on gold alloy containing 30\% silver electrodes of various roughnesses in aqueous perchlorate acid solution as supporting electrolyte in the absence and in the presence of $\mathrm{mM}$ of specifically adsorbed halide ions $\mathrm{X}\left(\mathrm{X}=\mathrm{Br}^{-}, \mathrm{Cl}^{-}, \mathrm{I}^{-}\right)$, at potentials where the dominant electrode process is the adsorption of the above anions. Efforts were mainly concentrated on the importance of surface preparation technique of the electrode and its influence on impedance spectra. Atomic scale inhomogeneities are introduced by mechanical treatment and can be decreased by annealing. Due to the annealing the double layer behaves as (almost) an ideal capacitance in the absence of specific adsorption though the surface roughness remains the same. A study of the related impedance behaviour in the presence of adsorbates even at very low concentrations $\left(10^{-4} \mathrm{M}\right)$, revealed capacitance dispersion increasing with the extent of specific anion adsorption at the gold/silver surface.
\end{abstract}

Keywords: metal/solution interface, impedance spectroscopy, cyclic voltammetry, capacitance dispersion, disorder, roughness, specific anion adsorption.

\section{Introduction}

The electrochemical interface that forms between electronically conducting media or electrodes (metals, semiconductors) and ionically conducting phases (electrolytes) plays a central role in many technologically important processes. Chemical and physical phenomena occurring at this interface are exploited in batteries and fuel cells, electroplating, sensors and catalysis.

The frequency dependence of the interfacial capacitance (or briefly the capacitance dispersion) has been observed quite often, even in the absence of

\footnotetext{
*Corresponding author. E-mail address: hammadi1@caramail.com
} 
faradaic reactions; the deviation from ideal capacitive behaviour is usually larger on rough electrodes than on smooth ones. The cause of frequency dispersion associated to impedance measurements is still a matter of controversy; thus, we start with a brief review of this problem. The first experiments revealing the role of surface roughness in causing capacitance dispersion were carried out by Borisova and Ershler [1]. Since Borisova-Ershler experiments, many papers have appeared with experimental results supporting the idea that capacitance dispersion is of purely geometrical origin $[2,3]$. The topic of rough electrodes became of interest when Mandelbrot's famous book [4] on fractals appeared. It was immediately suggested by a number of researchers [5, 6] that rough electrodes or porous surfaces could be modelled as fractals. The fractal (Hausdorf) dimension was connected to the so-called constant phase element, i.e., the frequency dispersion of the capacity, which is often used in impedance spectroscopy to fit the data.

This old early work in the mid-1980s on the fractal CPE models has used scaling arguments to explain this frequency dispersion. In these models, as a consequence, the spatial roughness range could not be converted to a temporal (or frequency) range within which the CPE behaviour is predicted on the basis of fractal geometry. Since than it became clear [7] that although the fractal CPE models are valid, under normal circumstances the fractal roughness-related CPE behaviour manifests itself in the high - frequency range ( $\mathrm{MHz}$ and above) which remains usually undetected in electrochemical impedance measurements. The work on rough capacitive electrodes [7-13] cleared up the issue, they showed, by giving experimental examples and counter-examples, that by choosing appropriate pre-treatment, cleaning procedures and electrode potential, almost ideal capacitive behaviour (CPE exponent of approx. 0.99) can be obtained even on rough surfaces. The reason for dispersion was shown to have kinetic rather than structural origin: $\mathrm{mM}$ quantities of $\mathrm{KCl}$ were shown to decrease the CPE exponent even on a smooth surface substantially, due to adsorption processes. Briefly, inhomogeneous surfaces give rise to a distribution of adsorption timeconstants. Ions adsorbed onto the surface contribute to the double-layer charge and their effects are inseparably coupled to the observable double-layer impedance. The distribution of adsorption time-constants thus leads to a distribution in the double layer/adsorption capacitance.

The modeling of the metal/electrolyte interface passes nowadays by the study of the topography of metal surfaces. In fact, polycrystalline solid surfaces are never perfectly flat, they comprise two topographic levels: microscopic and atomic. They are plane, neither on an atomic scale because of the existence of dislocations, nor on a broader scale because of the existence of grooves, undulations, scratches, etc. This is particularly true for a mechanically finished electrode. It is then necessary to distinguish between these two types of irregularities and one indicates the imperfections on an atomic scale as "disorder of surface" which refers to the presence at the same time of geometrical and energy inhomogeneousness which depends on the orientation and the crystalline defects; on the other hand, the term "roughness" is used exclusively for the geometrical imperfections of characteristic thicknesses of about $10 \mathrm{~nm}$. The 
surface disorder and roughness are often mutually dependent: mechanically rough surfaces increase a great number of dislocations.

In this paper we present experimental investigations using an integral technique such as impedance spectroscopy (EIS), based on "frequency dispersion capacitance", to reveal convincingly the importance of the electrode surface state and preparation of noble metal surfaces prior to electrochemical studies carried out with aqueous solutions, and to demonstrate that electrode preparation is a key issue in electrode kinetics; and, indeed, impedance spectroscopic results are related to atomic inhomogeneities instead of surface structural roughness. Furthermore, we demonstrate that specific ion adsorption plays a crucial role in capacitance dispersion; solvent molecules as well as adsorbed species can cause frequency dispersion through kinetic limitations such as mass transport (e.g. slow diffusion), adsorption, or phase transitions.

On the Au-Ag surface, we proceed to three types of tests.

1- Mechanical treatments to induce modifications of roughness.

2- Heat treatments to induce surface structural modifications.

3- Variation of the solutions and their ionic concentrations to induce a modification of behavior on the liquid side of the interface (adsorption).

The tests of impedances carried out then on Au-30\%Ag surfaces aim at evaluating the influence of these treatments and adsorption on the capacitive dispersion at the gold-silver metal interface.

The electrochemical methods described in this paper to investigate the electrochemical processes are well established in the electroanalytical field and include voltammetery and impedance spectroscopy. The latter technique provides a non-destructive method for investigating the electrochemical properties of the electrode, as all the data are obtained with a minimum polarization (i.e., change in the state of charge) of the electrode.

There are many electrode substances and preparation methods, and one can not suggest "the best" preparation technique. For studies with noble metals, the electrode preparation technology involves flame annealing, which yields clean, high quality surfaces. The surface pre-treatment procedure consists on roughening with emery paper (to make one rough electrode with a high level of atomic scale inhomogeneity) and applying a flame annealing (the mild annealing does not affect roughness but decreases the level of atomic scale inhomogeneity). The measurements were carried out in $0.1 \mathrm{M} \mathrm{HClO}_{4}$ containing dilute concentrations $\left(10^{-4} \mathrm{M}\right)$ of $\mathrm{KCl}, \mathrm{KBr}$ or $\mathrm{KI}$ to provide the $\mathrm{Cl}^{-}, \mathrm{Br}^{-}$or $\mathrm{I}^{-}$ions used as added anions in some experiments. All chemicals were of the highest purity available.

\section{Experimental \\ Experimental set-up}

The details of the experiments are as in [14]. The impedance spectra were measured by a standard electrochemical experimental set-up consisting of a commercial potentiostat (EG\&G PAR Model 183), and EG\&G InstrumentsPrinceton Applied Research 1025 Frequency Response Detector, both of which are connected to a controlling computer and custom software via an IEEE-488 
(GPIB) Interface Card. Commercially available control and measurement "Power Suite" ("Power Sine" electrochemical impedance and "Power CV" electrochemical voltammetery) software automates all of the subtasks involved in running the electrochemistry experiments. Directed by menu choices, it automatically controls the potentiostat, cell, and detector, acquires the data, and plots them on the screen. Cycling voltammetery and impedance measurements could be performed using the same set-up simply by changing the computer program. Prior to the measurements, the set-up and software was checked out with an ASTM dummy cell. Impedance spectra are acquired under potentiostatic EIS by maintaining under potentiostatic control the electrochemical cell in a two electrode configuration.

\section{Materials}

The electrochemical experiments were carried out with a conventional twoelectrode electrochemical cell made of pyrex glass of $10 \mathrm{~mL}$ of capacity manufactured by Glass-LAB-Constantine. The electrodes were circular disks of 1 $\mathrm{cm}^{2}$ of surface, $0.5 \mathrm{~mm}$ thick and spaced $1 \mathrm{~cm}$ apart from each other. The chemical compositions of the gold electrodes is $\mathrm{Au}-30 \% \mathrm{Ag}$, plunged in an aqueous solution of $0.1 \mathrm{M} \mathrm{HClO}_{4}$; for some measurements other chemicals of small $(\mathrm{mM})$ concentrations were added. In general high purity perchlorate acid and other chemicals (alkali-halide salts) of analytical grade were used. All measurements were taken at an ambient temperature of $\left(20 \pm 0.5^{\circ} \mathrm{C}\right)$. Impedance spectra were recorded in the frequency range $0.1 \mathrm{~Hz} \leq \mathrm{f} \leq 100 \mathrm{KHz}$.

To emphasize the extent of capacitance dispersion it is instructive to transform the $Z(\omega)$ impedance spectra to $C(\omega)$ complex capacitance by calculating $\mathrm{C}(\omega)=\frac{1}{[\mathrm{Z}(\omega)-\mathrm{Z}(\omega \rightarrow \infty)] \mathrm{j} \omega \mathrm{A}}$ function, where A stands for the geometric area of the electrode. The capacitance spectra, calculated from the impedances, were normalized to the same geometric electrode surface area using $1 \mathrm{~cm}^{2}$. Being a complex quantity, $\mathrm{C}(\omega)$ can be plotted in Bode representation. The extent of the capacitance dispersion is nil if the $\log \left(\mathrm{C}_{\mathrm{abs}}\right)$ vs. $\log (\mathrm{f})$ is a horizontal line and the phase angle, $\theta$, is zero everywhere. Increasing capacitance dispersion means more and more titled $\log \left(C_{a b s}\right)$ vs. $\log (f)$ curves and increasing $\theta$ values. An ideal CPE appears as straight line of slope $\alpha-1$ on the $\log \left(\mathrm{C}_{\mathrm{abs}}\right)$ vs. $\log (\mathrm{f})$ plot, and the phase angle is constant $\theta=90(1-\alpha)$. Any inaccuracy of the $R_{\text {sol. }}=Z(\omega \rightarrow \infty)$ value highly affects the accuracy of $C(\omega)$. This is the reason why the scatter of the high frequency points of the $\mathrm{C}(\omega)$ spectra are rather large.

\section{Results}

Prior to impedance spectroscopy measurements and in order to highlight the electrode current evolution as a function of potential, cyclic voltammetry measurements are made on an all-glass 3-electrodes electrochemical cell of cylindrical field distribution, manufactured by Glass-LAB-Constantine especially for this type of measurements. The gold working electrode is a cylindrical wire made using $0.4 \mathrm{~mm}$ diameter and $1 \mathrm{~cm}$ height, $\mathrm{Au}-30 \%$ polycrystalline $\mathrm{Ag}$ wire 
sealed in soft glass, forming a side contact surface area of $0.25 \mathrm{~cm}^{2}$; this geometry allows a homogeneous macroscopic current density distribution and a faster scan rate to be used for cyclic voltammetry. The counter-electrode is made of platinum wire spooled onto glass bars around the working electrode. All potentials quoted in this measurement are with respect to a calomel reference electrode saturated ( $\mathrm{SCE}$ ) (with saturated $\mathrm{KCl}$ filling solution). Better potential control is achieved using this 3-electrodes cell, in which the potential of the working electrode is controlled relative to the reference electrode, and the current passes between the working electrode and the third electrode (the auxiliary reference electrode).

The general cyclic voltammogram (cyclic voltammetry curve) response for the $\mathrm{Au}-\mathrm{Ag}$ electrode surface recorded in $0.1 \mathrm{M} \mathrm{HClO}_{4}$ solution at a sweep rate of 650 $\mathrm{mV} / \mathrm{s}$ versus SCE is shown in Fig. 1 and contains several characteristic regions: (a) a metallic region known as the double layer region, for which the current is solely due to double layer charging; it starts at potential $0.2 \mathrm{~V}$ versus SCE and corresponds to the potential where the metal/electrolyte interface is formed only by the charges orientation on both sides of the interface; (b) an oxide layer region (two clearly defined peaks at $0.2 \mathrm{~V}$ and $0.8 \mathrm{~V}$ vs. SCE) which corresponds to the formation of different oxide layers on the electrode surface (one generally uses a potential in the oxide layer region quite large, to ensure the formation of this layer, e.g. $\mathrm{E}=1.25 \mathrm{~V}$ ); (c) a well developed reduction peak of formed oxides ( $\mathrm{E}$ $=-0.2 \mathrm{~V}$ vs. SCE). The hysteresis between the anodic and cathodic peaks on the cyclic voltammogram arises from the electrochemical irreversibility of the oxide layer formation/dissolution; however, these processes and peaks are not important in the context of the present communication and, therefore, are further not mentioned.

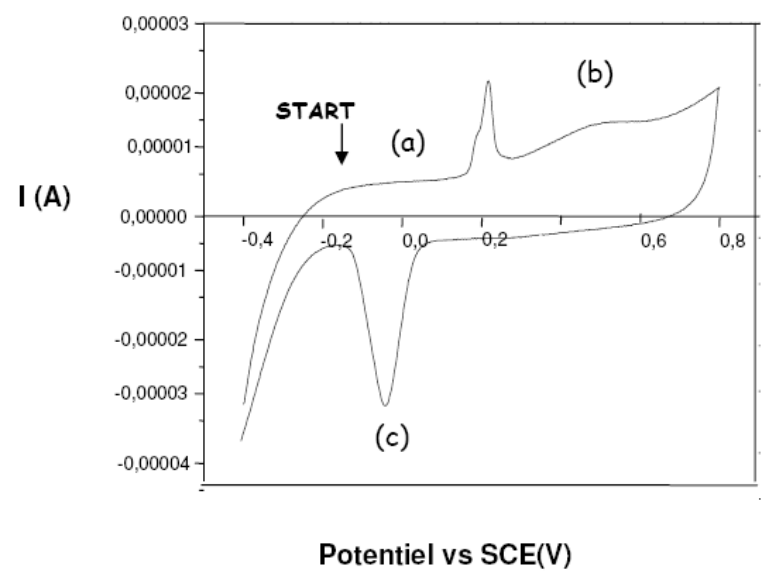

Figure 1. Cyclic voltammogram of $\mathrm{Au}-30 \% \mathrm{Ag}$ alloy electrode in $0.1 \mathrm{M} \mathrm{HClO}_{4}$ solution recorded at the sweep rate of $650 \mathrm{mV} / \mathrm{s}$ vs. SCE. The geometric area of the gold electrode is $0.25 \mathrm{~cm}^{2}$.

\section{Role of the nature of the surface}

On the Au-Ag electrodes, we proceed as follows $[11,13]$ :

The electrodes with surface defects were prepared by mechanical roughening of the electrode surface with an emery paper of grit 300 randomly. By this action, 
the gold-silver surface is roughened on microscopic scale while some surface defects are formed on atomic scale.

Clean electrodes should be used for the fabrication of working electrodes to assure that the only surface defects and roughness are those generated in the following process: the electrode was cleaned chemically by alternatively soaking in freshly made caroic acid (concentrated $\mathrm{H}_{2} \mathrm{SO}_{4}$ containing $\mathrm{H}_{2} \mathrm{O}_{2}$ ) and $10 \%$ $\mathrm{KOH}$ solution; it was then thoroughly rinsed by distilled water. Cleaning in such a way means that the electrode can safely be regarded as clean. This procedure of cleaning surfaces is of great importance, since the existence of any organic contaminations of surface defects can be the source of error. The capacitance of the $\mathrm{Au}-\mathrm{Ag}$ electrode measured in $0.1 \mathrm{M} \mathrm{HClO}_{4}$ solution at the oxide layer region $(\mathrm{E}=1.25 \mathrm{~V})$ (no effect of anion adsorption needs to be considered in this potential range; moreover, the perchlorate ion is only weakly specifically adsorbed on the polycrystalline gold-silver surface), showed a definite dispersion (Fig. 2, stars).

After this measurement, to prepare a defect-free gold/Ag electrode, the surface defects generated during the mechanical polishing were healed by thermal treatment. The best method for surface defect healing is flame annealing without any change on the structural roughness in microscopic scale. The roughened electrode was annealed in a Bunsen burner flame for 20 seconds. By this action, we have opportunity to compare two electrodes with exactly the same surface roughness but with and without surface defects.

The surface roughness of the roughened surfaces before and after thermal treatment was checked by means of technique of Scanning Electron Microscopy (SEM). No structural change in the surface roughness of the Au-Ag surface was observed; since such weak thermal treatment just heals atomic-scale imperfections, i.e. surface defects formed during mechanical polishing. After annealing, the electrode was rinsed in distilled water (a clean and a good quality surface should be obtained) and the impedance was again measured. The capacitance dispersion of the defect-free electrodes was found to be markedly decreased and this is illustrated in (Fig 2, triangles) and with a longer annealing, the capacitance spectrum of these electrodes further decreased (Fig. 2, circles) and approached almost ideal behavior. We were not able to achieve any further significant decrease of the dispersion by simple flame annealing.

We come to an important conclusion that a comparison of the results of impedance measurements at different steps of the procedure - roughening with emery paper, and flame annealing - reported in Fig. 2 obviously indicates the significant role of the surface defects appeared on the electrode surface, and that the existence of surface defects is a severe problem for fractal analysis by means of an electrochemical method such as impedance spectroscopy.

\section{Influence of specific anion adsorption}

It has been established experimentally that some anions in aqueous solutions are known for their great specific adsorption on nobles metals. Halides are by far the most exhaustively studied class of specifically adsorbing anions. However, the results of Horanyi $[15,16]$ have shown that the potential at which specific 
adsorption occurs varies from one species to the other. The chloride ions are most active in the potential interval $[0.1,1 \mathrm{~V}]$, the bromide ions are active in the interval $[-0.1,0.5 \mathrm{~V}]$ and the iodide ions are active in the interval [- 0.5, - $0.2 \mathrm{~V}]$. It should be noted here, that for adequate concentrations (of the order of $10^{-4} \mathrm{M}$ ), the process dominating at the electrode is adsorption. Dispersion is always more pronounced in the double layer region than in the oxide layer region and specific adsorption of these halide ions is suppressed in the oxide layer region $(E=1.25$ $\mathrm{V})$ thereby providing us with a "specific ion adsorption free reference state" of the gold-silver surface.

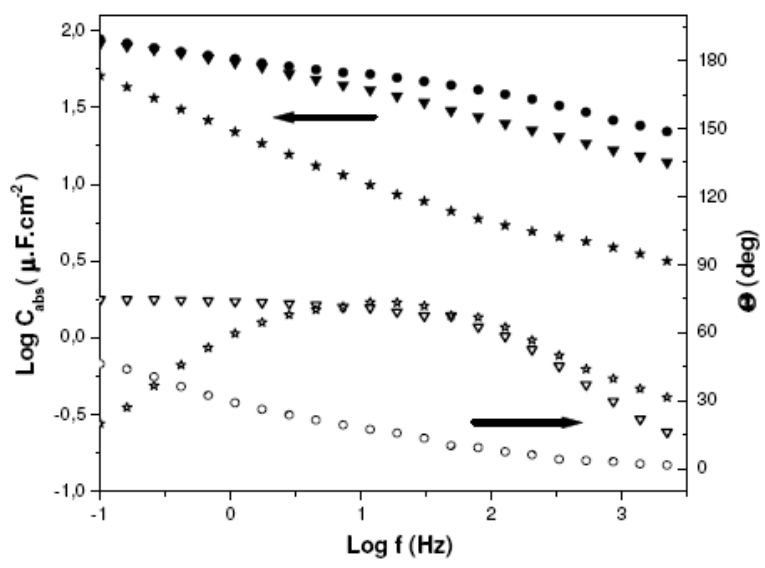

Figure 2. Capacitance spectra measured on $\mathrm{Au}-30 \% \mathrm{Ag}$ alloy electrode in the double layer region $(\mathrm{E}=-0.2 \mathrm{~V})$ in $0.1 \mathrm{M} \mathrm{HClO}_{4}$ base solution. Stars, original, roughened electrode with grit 300 emery paper; triangles, after a short annealing in Bunsen flame; circles, after a longer annealing. Full and open symbols refer to $\log C(\omega)$ and to $\theta$ values, respectively. Note that the capacitance is normalised with respect to the geometric surface area.

\section{a- Effects of concentration}

Impedance spectra of smooth clean (flame annealed surface) gold electrodes measured in the double layer region, at $-0.2 \mathrm{~V}$ in $0.1 \mathrm{M} \mathrm{HClO}_{4}$ containing added weak concentrations of $\mathrm{KBr}(0.001$ and $0.02 \mathrm{mM})$ are shown in Fig. 3. The choice of $\mathrm{KBr}$ is not fortuitous, the $\mathrm{Br}^{-}$are the least active vis-à-vis of the gold/silver surface. For comparison, a capacitance spectrum $(0 \mathrm{mM})$ measured in the absence of adsorption of bromide in the oxide layer region $(\mathrm{E}=1.25 \mathrm{~V})$ is also plotted. The capacitance dispersion increases markedly with the addition of bromide at concentrations as low as $0.001 \mathrm{mM}$ and $0.02 \mathrm{mM}$. We interpret the results of Fig. 3 as proof that capacitance dispersion is due to bromide adsorption.

\section{b) Effects of the species}

Impedance spectra of smooth clean (flame annealed surface) gold/silver electrodes measured in the double layer region, at $-0.2 \mathrm{~V}$ in $0.1 \mathrm{M} \mathrm{HClO}_{4}$ containing added $0.1 \mathrm{mM}$ of $\mathrm{KCl}, \mathrm{KBr}$, or $\mathrm{KI}$ are given in Fig. 4. For comparison a capacitance spectrum $(0 \mathrm{mM})$ measured in the absence of chloride in the oxide layer region $(\mathrm{E}=1.25 \mathrm{~V})$ is also plotted. 


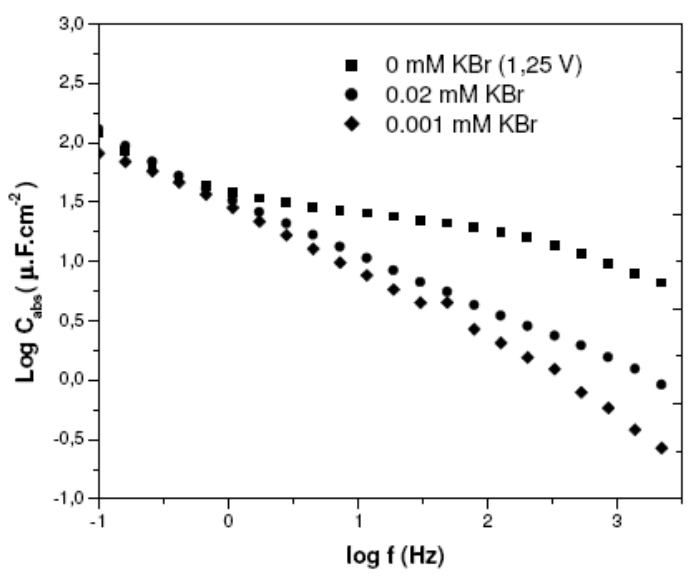

Figure 3. Capacitance spectra measured on $\mathrm{Au}-30 \% \mathrm{Ag}$ alloy electrode in the double layer region $(\mathrm{E}=-0.2 \mathrm{~V})$ in $0.1 \mathrm{M} \mathrm{HClO}_{4}$ containing added $\mathrm{Br}^{-}$of different concentrations. For comparison, a capacitance spectrum $(0 \mathrm{mM})$ measured in the absence of bromide in the oxide layer region $(\mathrm{E}=1.25 \mathrm{~V})$ is also plotted.

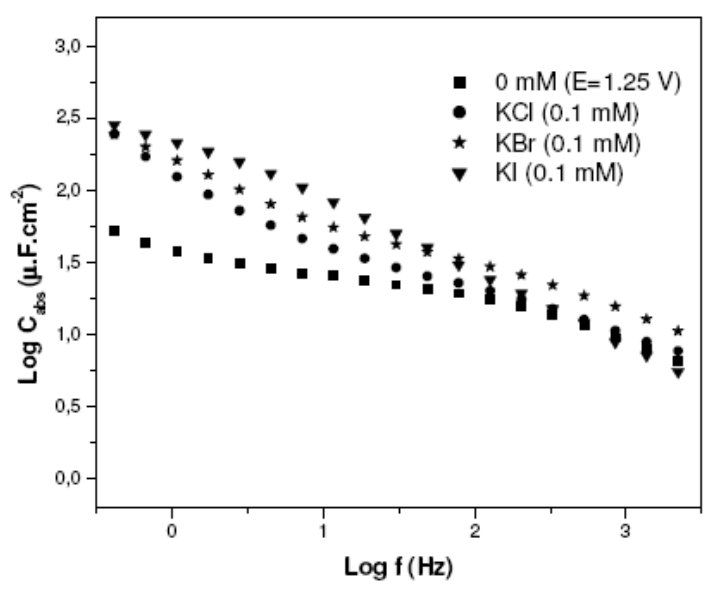

Figure 4. Capacitance spectra measured on $\mathrm{Au}-30 \% \mathrm{Ag}$ alloy electrode in the double layer region $(\mathrm{E}=-0.2 \mathrm{~V})$ in $0.1 \mathrm{M} \mathrm{HClO}_{4}$ containing added weak concentrations of $\mathrm{Cl}^{-}$, $\mathrm{Br}^{-}$or $\mathrm{I}^{-}$anions. For comparison a capacitance spectrum $(0 \mathrm{mM})$ measured in the absence of the above ions in the oxide layer region $(\mathrm{E}=1.25 \mathrm{~V})$ is also plotted.

Capacitance spectra show that the bromide, iodide or chloride addition, even in very low concentrations, increases capacitance dispersion very markedly. Taking into account that the more $\mathrm{Cl}^{-}, \mathrm{Br}^{-}$, and $\mathrm{I}^{-}$ions are specifically adsorbed, the larger their ionic radius (the estimated ability of the ions to approach the surface more closely is ascribed to their small size) we interpret the results as a proof that capacitance dispersion is due to the bromide, iodide or chloride adsorption. This quantity increases in the order $\mathrm{Cl}^{-}>\mathrm{Br}^{-}>\mathrm{I}^{-}$; we attribute this trend to the increase of the adsorption rate coefficient. 


\section{Conclusion}

The influence of preparation technique of the electrode surface and adsorption of the halide ions $\left(\mathrm{Cl}^{-}, \mathrm{Br}^{-}, \mathrm{I}^{-}\right)$at the aqueous perchlorate/Au-30\% $\mathrm{Ag}$ electrode interface have been studied by the impedance spectroscopy. The experimental results show a significant portion of capacitance dispersion observed when studying polycrystalline solid $\mathrm{Au}-30 \% \mathrm{Ag}$ electrodes which is due to atomic scale heterogeneities, and increases markedly if the solution contains added specifically adsorbing ions. More important is that electrochemical impedance spectroscopy based on "frequency dispersion of capacitance" is not reliable for fractal analysis of rough electrode surfaces in the presence of surface defects, since the calculated frequency dispersion depends on the defect density of the surface; the presence of such surface defects can make errors in impedance spectroscopy measurements.

\section{References}

1. T.I. Borisova and B.V. Ershler, Zh. Fiz. Khim. 25 (1950) 337.

2. $\quad$ R. de Levie, Electrochim. Acta 10 (1965) 113.

3. W. Scheider, J. Phys. Chem. 79 (1975) 127.

4. B. Mandelbrot, Les objets fractals, Flammarion, Paris, 1975.

5. A. Le Mehauté, A. De Guibert, M. Delaye, C. Flippi, C.R. Acad. Sci. (1982) 835.

6. L. Nykos, T. Pajkossy, Electrochim. Acta (1985) 1533.

7. T. Pajkossy, J. Electroanal. Chem. (1994) 111.

8. T. Pajkossy, Heterogeneous Chem. Rev. 2 (1995) 143.

9. T. Pajkossy, T. Wandlowski, D.M. Kolb, J. Electroanal. Chem. 414 (1996) 2093.

10. T. Pajkossy, Solid State Ionics 94 (1997) 123.

11. Z. Kerner, T. Pajkossy, J. Electroanal. Chem. 448 (1998) 139.

12. T. Pajkossy, D.M. Kolb, Electrochim. Acta 46 (2001) 3063.

13. Z. Kerner, T. Pajkossy, Electrochim. Acta 47 (2002) 2055.

14. A. Hammadi, M. Berd, Portugaliae Electrochim. Acta 23 (2005) 437.

15. G. Horanyi, J.Solt, F. Nagy, J. Electroanal. Chem. 31 (1971).

16. G. Horanyi, Electrochim.Acta 25 (1980) 1359. 